\title{
Clinical Features and Efficacy of Antiviral Drug, Arbidol in 220 Nonemergency COVID-19 Patients from East-West-Lake Shelter Hospital in Wuhan: A Retrospective Case Series
}

\section{Wei Gao}

Shanghai East Hospital

Si Chen

Shanghai East Hospital

Kun Wang

Shanghai East Hospital

Rongzhang Chen

Shanghai East Hospital

Qian Guo

Shanghai East Hospital

Jingjing Lu

Shanghai East Hospital

Xiaodong Wu

Shanghai East Hospital

Yanan He

Shanghai East Hospital

Qiaoyun Yan

Shanghai East Hospital

Shengyun Wang

Changhai Hospital

Feilong Wang

Shanghai East Hospital

Li Jin

Shanghai East Hospital

Jing Hua

Shanghai East Hospital

Qiang Li (D871242050@qq.com)

Shanghai East Hospital 


\section{Short report}

Keywords: Coronavirus disease, COVID-19, Arbidol, Efficacy, SARS-CoV-2

Posted Date: September 11th, 2020

DOl: https://doi.org/10.21203/rs.3.rs-38631/v2

License: (c) (i) This work is licensed under a Creative Commons Attribution 4.0 International License. Read Full License

Version of Record: A version of this preprint was published at Virology Journal on October 23rd, 2020. See the published version at https://doi.org/10.1186/s12985-020-01428-5. 


\section{Abstract}

Objective. We aimed to describe the features of 220 nonemergency (mild or common type) COVID-19 patients from a shelter hospital, as well as evaluate the efficiency of antiviral drug, Arbidol in their disease progressions.

Methods. Basic clinical characteristics were described and the efficacy of Arbidol was evaluated based on gender, age, maximum body temperature of the patients.

Results. Basically, males had a higher risk of fever and more onset symptoms than females. Arbidol could accelerate fever recovery and viral clearance in respiratory specimens, particularly in males. Arbidol also contributed to shorter hospital stay without obvious adverse reactions.

Conclusions. In the retrospective COVID-19 cohort, gender was one of the important factors affecting patient's conditions. Arbidol showed several beneficial effects in these patients, especially in males. This study brought more researches enlightenment in understanding the emerging infectious disease.

\section{Introduction}

Since December 2019, an outbreak of unexplained epidemic pneumonia occurred in Wuhan, Hubei Province, China and has soon spread to the whole country. As of 6 May 2020, it spread to other 215 countries and a total of 3, 721, 393 globally laboratory-confirmed cases have been reported. On 11 February 2020, the novel epidemic disease was formally named as coronavirus disease 2019 (COVID-19) and its causative virus as severe acute respiratory syndrome coronavirus 2 (SARS-CoV-2) [1]. In China, the number of COVID-19 infections has exceeded that of SARS population in 2002. In order to prevent the rapid spread of COVID-19, the Chinese government established 16 shelter hospitals for nonemergency patients (mild and common type). Within 35 days, Chinese doctors in these hospitals cured more than 10, 000 patients with a zero death. However, we haven't fully understood the clinical characteristics, disease evolution and therapeutic regime of COVID-19 patients in the special hospitals.

For the diagnosis of COVID-19, viral nucleic acid assay played a vital role by use of oropharyngeal swabs samples [2]. Comparatively, effective antiviral therapies seemed uncertain [2]. Arbidol, Oseltamivir, Ribavirin, Lopinavir/Ritonavir and Interferon (individual or combined treatment) were widely used in COVID-19 patients around China; however, none of them exhibited significant efficacy. We speculated that the results might partly attribute to the unclassified population under the tense epidemic situation. Herein, we described the features of 220 relatively mild COVID-19 patients from a shelter hospital, as well as evaluated the therapeutic efficiency of an antiviral drug, Arbidol.

\section{Methods}

Our retrospective cohort from the East-West-Lake shelter hospital was composed of 220 laboratoryconfirmed COVID-19 patients from 12 January 2020 to 2 March 2020. Approval for the retrospective 
analysis was obtained from the Ethics Commission of Shanghai East Hospital, China. The privacy rights of human subjects were protected all long.

The clinical features of different therapeutic groups were exhibited in Table1 and Table2. A number of 130 patients received oral Arbidol at a dosage of $200 \mathrm{mg}, 3$ times a day for 4-8 days. Among them, 40 patients were given Arbidol and other antiviral drugs, including Oseltamivir ( 39 cases, $150 \mathrm{mg}, 2$ times a day for 4-8 days)/Ribavirin (1 case, $500 \mathrm{mg}, 2$ times a day for 4-8 days)) while others were treated with Arbidol only. Among other 90 patients, 45 haven't used any antiviral drugs and the remains were administrated with other antiviral drugs, including Oseltamivir (43 cases), Ribavirin (1 case), or Ganciclovir (1 case, $500 \mathrm{mg}, 2$ times a day for 4-8 days).

For COVID-19 diagnosis, oropharyngeal swabs samples were collected for viral nucleic acid assay. The detection of SARS-CoV-2 nucleic acids in respiratory specimens was based on the final result and time. If the last two assays at an interval of at least 24-hour were both negative, the viral nucleic acid negative conversion time was calculated using the previous one; otherwise, we considered it as not negative (including positive and suspected). Statistical analysis was performed using SPSS 17.0. Appropriate statistical methods were applied according to different data types.

\section{Results}

Firstly, we described general characteristics of the retrospective cohort (Table 1 and 2). They were mild type (1 case) or common type (219 cases) COVID-19 patients according to the updated guidance [2]. Among them, male patients had a higher risk of fever than females (odds ratio $(O R)=2.47,95 \%$ confidence interval $(\mathrm{Cl}): 1.25-4.89, \mathrm{p}=0.01)$. They also tended to get more onset symptoms $(\geq 3)$ than women (OR=1.88, 95\% Cl:1.08-3.27, $\mathrm{p}=0.03)$ (Table S1). Based on the analysis, we concluded that gender might be a significant influence factor and should be taken into account when assessing the efficacy of Arbidol in the non-severe COVID-19 cohort.

Afterwards, we found that fever resolved more slowly in patients without Arbidol administration (hazard ratio $(\mathrm{HR})=0.69,95 \% \mathrm{Cl}: 0.48,0.99, \mathrm{p}=0.02)$ (Figure $1 \mathrm{~A})$. When subgrouping by therapeutic strategies, we only discovered the significant improvement of recovery time between other antiviral drugs $(91.2 \%$ Oseltamivir) group and Arbidol group ( $\mathrm{HR}=0.58,95 \% \mathrm{Cl}: 0.37-0.92, \mathrm{p}=0.02)$ (Figure 1B-E). Combination of Arbidol and other antiviral drugs ( $97.5 \%$ Oseltamivir) did not show better efficacy (Figure 1E). Besides, in males and patients with lower-grade fever $\left(\leq 38.5^{\circ} \mathrm{C}\right)$, Arbidol showed superior efficacy in fever recovery $(\mathrm{HR}=0.59,95 \% \mathrm{Cl}: 0.37-0.95, p=0.03 ; \mathrm{HR}=0.57,95 \% \mathrm{Cl}: 0.34-0.95, p=0.03)$, which were not obvious in women and higher-grade fever subjects (Figure 2A-D). Age was not the key point that affected Arbidol efficacy in this respect (Figure 2E-F).

Subsequently, we observed that negative-converting rate of nucleic acid within 14 days in non-Arbidol group was lower than that of Arbidol group (OR=0.47, 95\% Cl:0.24-0.91, $\mathrm{p}=0.028)$. The effect of Arbidol 
was more remarkable when compared to patients without any antiviral drugs $(\mathrm{OR}=0.23,95 \% \mathrm{Cl}: 0.10-0.57$, $\mathrm{p}=0.002$ ). At the last assay, a total number of 14 patients still got non-negative results. In patients without Arbidol application, we saw higher non-negative rate compared with others (OR=3.13, 95\% Cl:1.00-9.83, $\mathrm{p}=0.049$ ) (Table 3). Consistent with the above data, Arbidol showed obvious efficacy on viral clearance in males (OR=0.27, 95\% Cl:0.11-0.66, $p=0.005$ for negative-conversion within 2 weeks; OR=8.40, 95\% Cl:1.70$41.42, p=0.006$ for not negative rate at last assay). In particular, we found that patients with not negative results in non Arbidol group were all males, which was improved noticeably in Arbidol group (Table 4).

The medium hospital day in patients without antiviral drugs and treated with Arbidol was 19 and 15.5, respectively $(p=0.02)$. Considering the influence factors, we further demonstrated that Arbidol might contribute to the reduced hospitalization times in younger patients ( $\leq 50$ year, $p=0.04)$ (Table 5$)$. During our observation period, no obvious adverse reaction was noted in Arbidol treated patients. One case from Arbidol group presented with allergic skin rash due to Moxifloxacin and the medication had to be discontinued.

\section{Discussion}

Arbidol was a broad-spectrum antiviral agent developed 30 years ago and has been reported to have inhibitory effects on a diverse array of viruses such as influenza, Zika virus, respiratory syncytial, adenovirus, Coxsackie B5, parainfluenza, Ebola and hepatitis B and C [3-7]. Mechanismly, Arbidol might impair several steps within the life cycle of viruses, including attachment to cells, fusion with cellular membranes during virus entry. More precisely, it inhibited the fusion of influenza virus with endosomal membrane through binding to a hydrophobic cavity in the hemagglutinin on virus surface and stabilizing the prefusion conformation of hemagglutinin [5]. Owing to the broad-spectrum efficacy, Arbidol has been licensed for prophylaxis and treatment of acute respiratory infections, including influenza in China and Russia [5]. As for COVID-19, Lu et al. [8] found no difference between Lopinavir/Ritonavir and Arbidol in relieving symptoms or accelerating virus clearance. However, the subsequent multicenter, prospective research carried out by Li.et.al [9] demonstrated that the triple combination antiviral therapy of Arbidol, Lopinavir/Litonavir and recombinant interferon $\mathrm{a}-2 \mathrm{~b}$ showed shorter viral shedding time and hospitalization time compared with the dual combination antiviral therapy without Arbidol. They also found that 10-30 $\mu \mathrm{mol} / \mathrm{L}$ Arbidol effectively inhibited the coronavirus sixty-fold compared with the untreated control group, as well as significantly alleviated the injury of SARS-CoV-2 to cells by chemosensitivity testing in vitro (Data were not published). Herein, we discovered the efficacy of Arbidol on viral shedding, thus accelerating disease relief in the nonemergency COVID-19 patients. We noticed that males displayed higher fever and more COVID-19 symptoms, which might due to the up-regulated SARS-Cov-2 receptor, angiotensin-converting enzyme 2 (ACE2) by smoking and testosterone level, as well as excessive immune-inflammatory response $[10,11]$. Furthermore, males exhibited better drug reactions, suggesting certain microenvironment (such as $\mathrm{pH}$, ion, hormone and cytokines) might strengthen the efficacy of Arbidol. More studies in vivo and in vitro could be performed to identify the exact mechanisms. 
Several results deserved explanations. Arbidol shortened fever duration compared with the patients without Arbidol and with other antiviral drugs respectively (Figure 1 and 2). The effect seemed more prominent when given early in the disease and in male patients. As shown in Figure 1E, combination of Arbidol and other antiviral drugs did not show better efficacy compared to Arbidol only. We speculated the reduction in the first 5 days of fever period was mainly due to Arbidol and application of several antiviral drugs simultaneously aggravated adverse reaction or induced multiple adverse reactions. Additionally, all patients in Arbidol + other antiviral drugs group achieved negative nucleic acid in their respiratory specimens, but not in Arbidol only group (Table 3), though the difference was insignificant. We suggested that the number of subjects was not enough, therefore leading to certain contingency in the result.

Despite efforts to exclude bias by critical analysis, there were still several limitations in our research. First of all, this was a retrospective study and has not undergone rigorous clinical trial design. Therefore, it could not provide direct evidence for the effectiveness of Arbidol among COVID-19 patients. However, these results provided implications for further experimental or clinical researches on Arbidol usage and even guided the development of novel therapeutics against SARS-CoV-2. Secondly, due to the early onset (from January to February, 2020) of the disease in this cohort and undetectable influenza assay, the treatment therapies might not accord with the latest guideline. These relatively mild patients were mainly administrated with Arbidol and/or Oseltamivir as antiviral treatments because of the rescuing urgency. In that case, we excluded the bias of antibiotic and traditional Chinses medicine which were widely used in our cohort (Table 1 and Table 2) and found the significant results of Arbidol. It should be noted that the efficacy might attribute to the combined effects, such as Arbidol combined with Chinses medicine. This deserved further studies in a larger cohort. Thirdly, the patients were hospitalized in shelter hospital (originated from gymnasiums, convention center and so on), where laboratory tests and chest CT could not be carried out promptly as a consequence of equipment and faculty deficiency. Therefore, our efficacy evaluation system was not perfect. They could not be performed viral nucleic acid detection everyday nor within 7 days since the first diagnosis; thus, we selected negative-conversion within 14 days as the indication of better drug response. This study aimed to bring more researches enlightenment in understanding the emerging infectious disease. Despite this, we also suggested the double-blinded randomized clinical trials on Arbidol application in COVID-19 patients, especially in mild and common type.

\section{Conclusion}

In the retrospective COVID-19 cohort of 220 nonemergency patients form one shelter hospital, we analyzed and concluded that male patients had a higher risk of fever and more onset symptoms than females. Besides, Arbidol showed beneficial effects on fever recovery, viral clearance and shorter hospital stay in these patients, especially in males. Double-blinded randomized clinical trials to determine the most effective treatments for COVID-19 are still needed. Finally, we hope that human beings can soon overcome difficulties together in the "war" against COVID-19. 


\section{Abbreviations}

COVID-19: coronavirus disease 2019

SARS-CoV-2: severe acute respiratory syndrome coronavirus 2

OR: odds ratio

Cl: 95\% confidence interval

HR: hazard ratio

Angiotensin-converting enzyme 2: ACE2

\section{Declarations}

\section{Ethics approval and consent to participate}

The study was approved by Shanghai East Ethics Committee. Written informed consent was waived by the Ethics Committee due to the retrospective nature of this study and rapid emergence of this infectious disease.

\section{Consent to publication}

All co-authors have approved the manuscript and agreed with the publication. This manuscript did not contain any individual person's data in any form.

\section{Availability of data and material}

The datasets used and/or analyzed during the current study are available from the corresponding author on reasonable request.

\section{Competing interests}

The authors declare no competing interests.

\section{Funding}

This work was supported by National Natural Science Foundation of China (grant number: 81870064), "Gaoyuan" project of Pudong Health and Family Planning Commission (grant number: PWYgy2018-06), National Key R\&D Program (grant number: 2018YFC1313700) from Qiang Li and the Research Foundation of Shanghai Science and Technology Commission (grant number: 18140904100) from Shengyun Wang.

\section{Authors' contributions}


JH conceived, designed the study. WG, SC and KW analyzed the data and wrote the paper. RC, QG, JL, XW, YH, QY, SW, FW and LJ contributed to data acquisition and analysis. QL interpreted the data and put expert insights in this study.

\section{Acknowledgements}

We acknowledge all the frontline workers in the city of Wuhan for their remarkable efforts to provide care for the critically ill patients with COVID-19.

\section{References}

1] Guan WJ, Ni ZY, Hu Y, Liang WH, Ou CQ, He JX, et al. Clinical Characteristics of Coronavirus Disease 2019 in China. N Engl J Med. 2020;382:1708-20.

[2] National Health Commission of the People's Republic of China. Pneumonia diagnosis and treatment for COVID-19 infection (trial version 7 revised version) [EB/OL]. (2020-03-03) [2020-03-04]. http://www.nhc.gov.cn/xcs/zhengcwj/202003/46c9294a7dfe4cef80dc7f5912eb1989.shtml.

[3] Hulseberg CE, Feneant L, Szymanska-de Wijs KM, Kessler NP, Nelson EA, Shoemaker CJ, et al. Arbidol and Other Low-Molecular-Weight Drugs That Inhibit Lassa and Ebola Viruses. J Virol. 2019;93:e02185-18.

[4] Fink SL, Vojtech L, Wagoner J, Slivinski NSJ, Jackson KJ, Wang R, et al. The Antiviral Drug Arbidol Inhibits Zika Virus. Sci Rep. 2018;8:8989.

[5] Kadam RU, Wilson IA. Structural basis of influenza virus fusion inhibition by the antiviral drug Arbidol. Proc Natl Acad Sci U S A. 2017;114:206-14.

[6] Fediakina IT, Leneva IA, lamnikova SS, Livov DK, Glushkov RG, Shuster AM. Sensitivity of influenza $\mathrm{A} / \mathrm{H} 5$ viruses isolated from wild birds on the territory of Russia to arbidol in the cultured MDCK cells. Vopr Virusol. 2005;50:32-5.

[7] Boriskin YS, Leneva IA, Pecheur El, Polyak SJ. Arbidol: a broad-spectrum antiviral compound that blocks viral fusion. Curr Med Chem. 2008;15:997-1005.

[8] Chen J, Ling Y, Xi X, Liu P, Li F, Li T, et al. Efficacies of lopinavir/ritonavir and abidol in the treatment of novel coronavirus pneumonia. Chin J Infect Dis. 2020;38:E008.

[9] Wei R, Zheng N, Jiang X, Ma C, Xu X, Liu S, et al. Early antiviral therapy of abidor combined with lopinavir/ritonavir and re-combinant interferon $a-2 b$ in patients with novel coronavirus pneumonia in Zhejiang: A multicenter and prospective study. Chin J Clin Infect Dis. 2020;13: 9-15.

[10] Jacobs M, Van Eeckhoutte HP, Wijnant SRA, Janssens W, Joos GF, Brusselle GG, et al. Increased expression of ACE2, the SARS-CoV-2 entry receptor, in alveolar and bronchial epithelium of smokers and COPD subjects. Eur Respir J. 2020;56:2002378. 
[11] Giagulli VA, Guastamacchia E, Magrone T, Jirillo E, Lisco G, De Pergola G, et al. Worse progression of COVID-19 in men: Is testosterone a key factor? Andrology. 2020:10.1111/andr.12836.

\section{Tables}

Due to technical limitations, the tabes are provided in the Supplementary Files section.

\section{Figures}



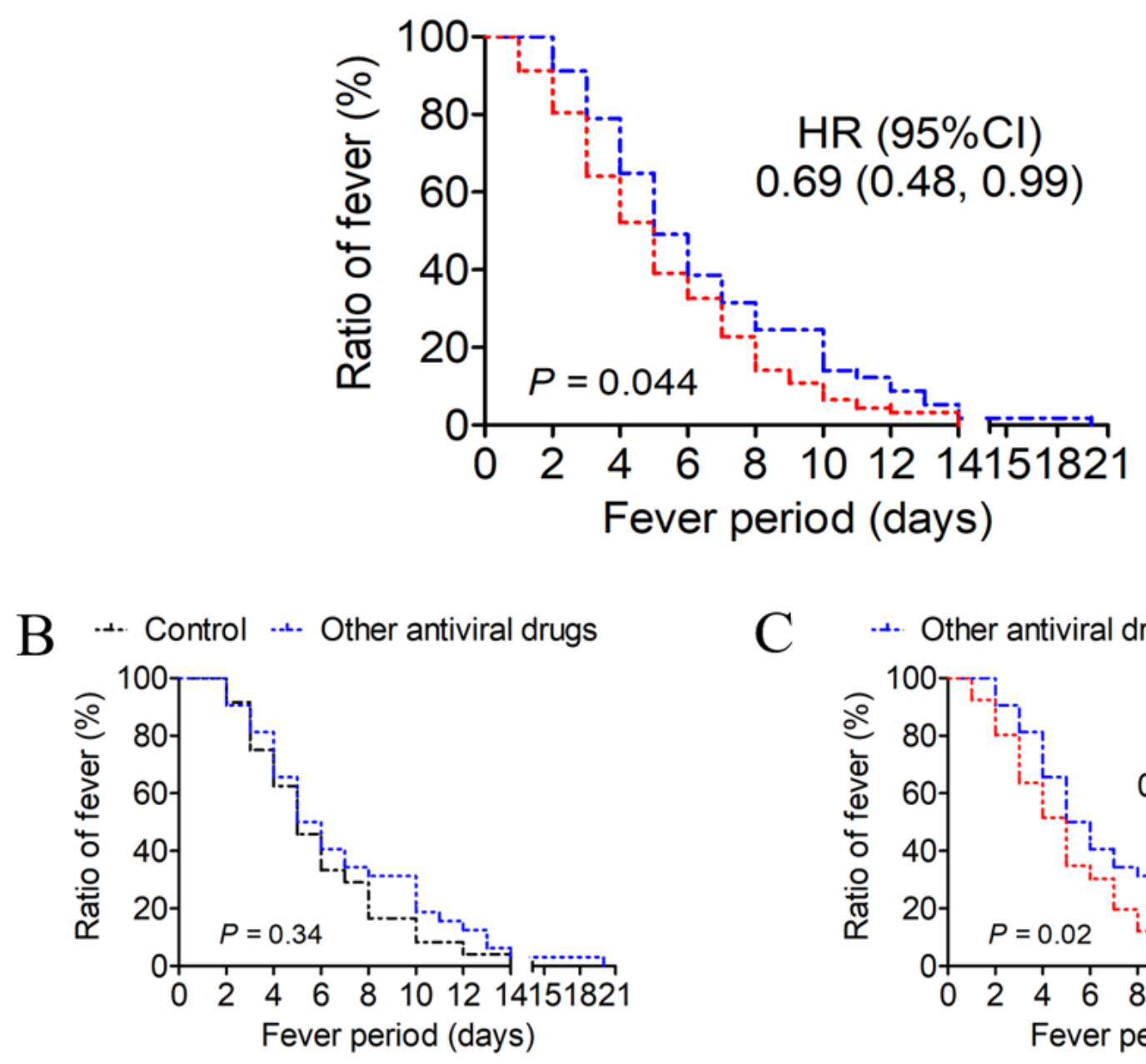

C ... Other antiviral drugs .... Arbidol
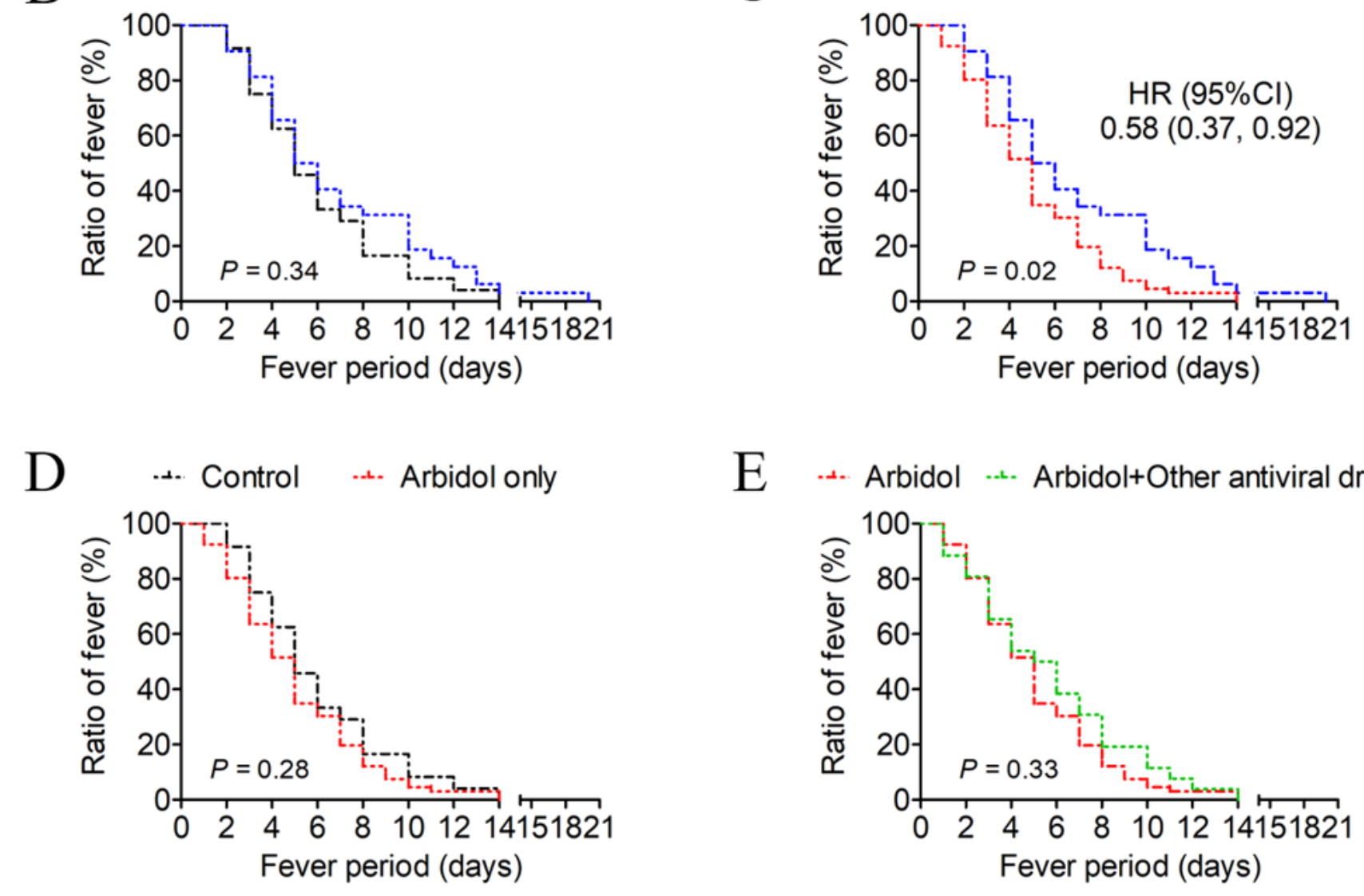

\section{Figure 1}

Time to fever resolution compared between distinct group based therapeutic strategies: $(A)$ with and without Arbidol, (B) control and other antiviral drugs, (C) other antiviral drugs and Arbidol, (D) control and Arbidol only, (E) Arbidol and Arbidol combined with other antiviral drugs. HR (95\% Cl): hazard ratio (95\% confidence interval). 
A

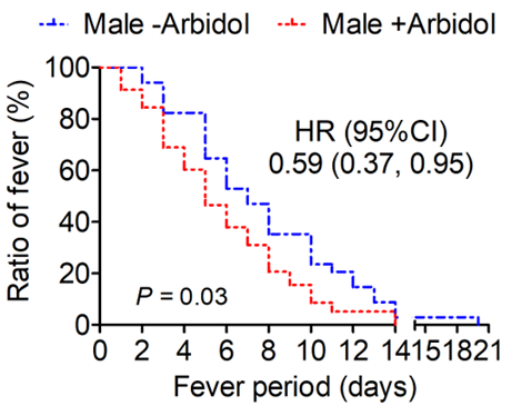

B ... Female -Arbidol -... Female +Arbidol

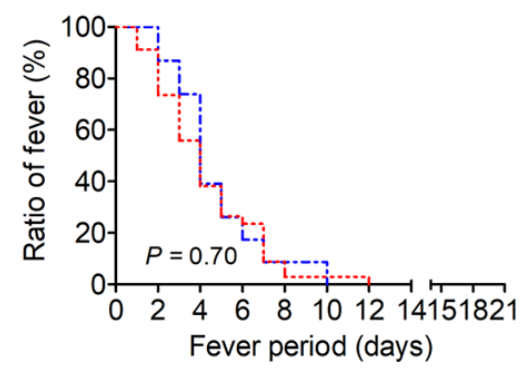

C

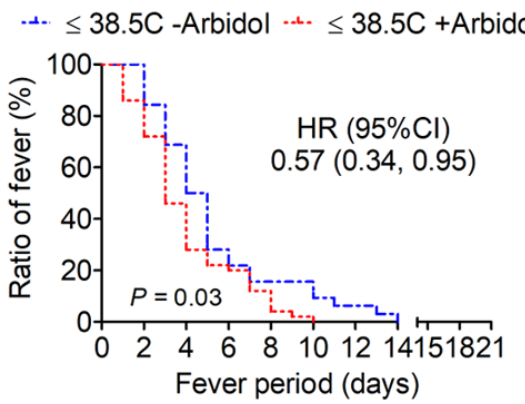

$\mathrm{D}$

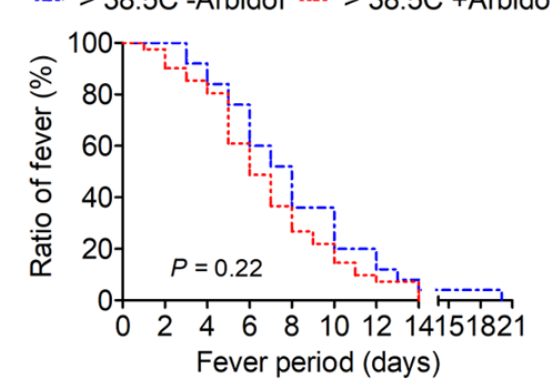

E

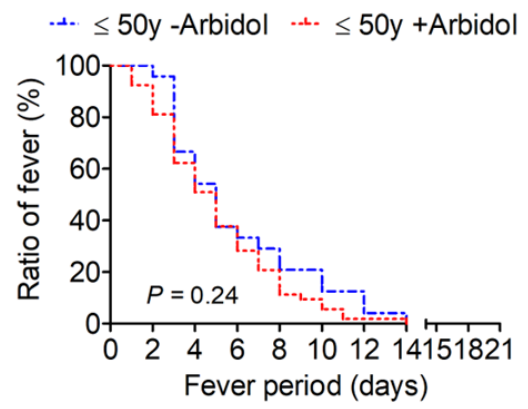

$\mathrm{F}$

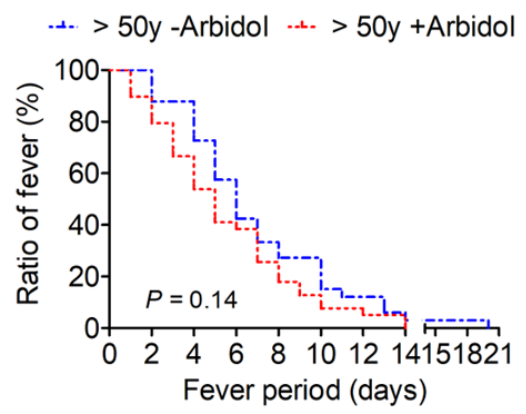

\section{Figure 2}

Time to fever recovery compared between distinct group based on gender (A, B), maximum body temperature $(C, D)$ and age $(E, F)$. HR $(95 \% C l)$ : hazard ratio (95\% confidence interval).

\section{Supplementary Files}

This is a list of supplementary files associated with this preprint. Click to download.

- Table1.tif

- Table2.tif

- Table3.tif

- Table4.tif

- Table5.tif

- Tables1.tif 